\section{Mapeamento das preferências de atores estratégicos sobre os critérios de priorização para o monitoramento do horizonte tecnológico em saúde}

\author{
Mapping stakeholders' preferences in prioritization \\ criteria for horizon scanning in healthcare \\ technologies
}

Mapeo de las preferencias de actores estratégicos sobre los criterios de priorización en la evaluación de tecnologías emergentes en salud

\section{Resumo}

Entre as etapas básicas de um sistema de monitoramento do horizonte de tecnologias emergentes, estão a filtragem e a priorização dessas tecnologias. Este trabalho tem por objetivo mapear as preferências dos atores estratégicos do Sistema Único de Saúde (SUS) quanto aos critérios de filtragem e priorização. Foram selecionados dois critérios de filtragem (horizonte de tempo e inovação) e oito critérios de priorização (relevância: epidemiológica, nas políticas de saúde e na prática clínica; impacto: orçamentário no SUS, no custo para o serviço em saúde e na mortalidade; segurança, e aspectos legais, éticos e sociais). A análise de correspondência múltipla foi aplicada para mapear as preferências dos atores nos grupos e entre grupos. Dois grupos foram mais homogêneos entre si e determinantes para a seleção dos critérios de priorização. A influência da formação profissional foi maior que a institucional. A metodologia permitiu a seleção de critérios de forma transparente e a análise das preferências individuais dos participantes.

Tecnologia Biomédica; Avaliação da Tecnologia Biomédica; Planejamento em Saúde
Aline do Nascimento 1

Avila Teixeira Vidal 2

Rosimary Terezinha de Almeida 1
1 Instituto Alberto Luiz
Coimbra de Pós-graduação
e Pesquisa de Engenharia, Universidade Federal do Rio
de Janeiro, Rio de Janeiro,
Brasil.
2 Secretaria de Ciência
Tecnologia e Insumos
Estratégicos, Ministério da
Saúde, Brasília, Brasil.

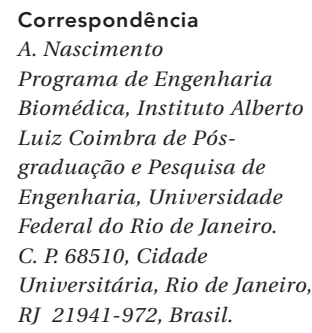




\section{Introdução}

O processo de incorporação de tecnologias em saúde no Brasil tem passado por mudanças significativas nos últimos anos. Apesar dos avanços alcançados, esse processo ainda é reativo devido às dificuldades de prospectar tecnologias relevantes para o Sistema Único de Saúde (SUS). Esse cenário se torna ainda mais complexo ao considerar o grande volume de tecnologias novas no mercado (em fase de aprovação ou recém-aprovadas pela agência reguladora) e emergentes (em fase de pesquisa clínica) ${ }^{1}$. Assim, é fundamental a presença de informação consistente e em tempo hábil sobre essas tecnologias para tornar o processo de incorporação proativo.

Essa necessidade faz surgir uma etapa específica na Avaliação de Tecnologia em Saúde (ATS) denominada Monitoramento do Horizonte Tecnológico (MHT) 1,2. O MHT tem o objetivo de identificar tecnologias novas e emergentes e antecipar os impactos que elas possam causar no sistema de saúde, assim como disseminar informações que sejam relevantes para os tomadores de decisão ${ }^{3}$. Nesse sentido, o MHT pode auxiliar o processo de incorporação, antecipando as demandas e a identificação das tecnológicas mais relevantes para o SUS.

Não existe uma forma única de desenvolver o MHT, pois o processo vai depender dos objetivos a serem alcançados e para que tipo de cliente as informações se destinam. Contudo, existe uma diretriz internacional para realizar o MHT, desenvolvida pela European Information Network on New and Emerging Health Technlogies (Euro Scan) ${ }^{3}$. A EuroScan é uma rede composta por 18 agências de ATS que têm por objetivo a troca de informações e o desenvolvimento de métodos para a identificação e avaliação precoce sobre tecnologias novas e emergentes 4 .

A diretriz da EuroScan tem como etapas iniciais: identificação do cliente que utilizará a informação; determinação do horizonte de tempo, que definirá a fase do ciclo de vida da tecnologia a ser prospectada; e escolha do tipo de tecnologia. Tais etapas são fundamentais na definição dos critérios de filtragem e de priorização, que irão definir as tecnologias candidatas a serem monitoradas. Uma vez que as tecnologias candidatas foram identificadas, as etapas seguintes são: avaliação, disseminação e atualização dos informes, quando for necessário 3 .

No Brasil, a preocupação inicial com as ações de MHT ocorreu no âmbito da Rede Brasileira de Avaliação de Tecnologia em Saúde (REBRATS), em 2008. Uma das primeiras atividades foi a realização em 2010 da oficina de Monitoramento do Horizonte Tecnológico no III Seminário de
Gestão de Tecnologias em Saúde, em Salvador, Bahia 5. Participaram dessa oficina gestores dos órgãos do Ministério da Saúde e das agências reguladoras (Agência Nacional de Saúde Suplementar - ANS e Agência Nacional de Vigilância Sanitária - ANVISA); provedores de serviços de saúde; pesquisadores e especialistas em avaliação de tecnologias em saúde. A oficina teve por objetivo a elaboração de uma proposta para estabelecer um sistema de MHT no SUS, tendo como base a diretriz da EuroScan 5 .

Como principais resultados dessa oficina, foi definido como cliente prioritário a Comissão de Incorporação de Tecnologias do Ministério da Saúde (CITEC), atualmente denominada como Comissão Nacional de Incorporação de Tecnologias no SUS (CONITEC), que tem a finalidade de assessorar o Ministério da Saúde na incorporação, exclusão ou alteração de tecnologias em saúde no SUS 6 . Outro aspecto consensual foi que as tecnologias candidatas deveriam atender aos problemas prioritários de saúde do país, independentemente do tipo de tecnologia 5 .

Esses resultados foram discutidos posteriormente no âmbito da REBRATS, sendo apresentadas pelo cliente (CONITEC) as áreas cardiovascular e oncológica como áreas prioritárias para a prospecção de tecnologias novas e emergentes. No entanto, ainda não foi definida a sistemática das etapas do processo de MHT para que seja adotado e implementado no SUS.

Dessa forma, este estudo teve por objetivo propor uma metodologia para mapear as preferências de atores estratégicos do SUS sobre os critérios de priorização de um sistema de MHT em saúde. Para contextualizar a aplicação da metodologia, foi escolhido o cenário de medicamentos oncológicos, uma das áreas definida como prioritária para a prospecção de tecnologias novas e emergentes no SUS.

\section{Materiais e métodos}

Esta seção está dividida nos passos gerais realizados para a construção do estudo, que foram: identificação na literatura dos critérios de filtragem e priorização, seleção dos critérios de filtragem e priorização e análise das preferências dos atores estratégicos na incorporação de tecnologia em saúde.

\section{Identificação dos critérios de filtragem e priorização}

Os critérios propostos na definição da metodologia foram selecionados por uma pesquisa nos sítios eletrônicos de cada agência membro 
da EuroScan 4 e no suplemento especial da International Journal of Technology Assessment in Health Care, que fez uma retrospectiva internacional sobre o tema "Early awareness and alert (EAA) methods and systems" 7. Esses critérios foram discutidos em reunião prévia com o cliente CONITEC por meio de um representante do Departamento de Gestão e Incorporação de Tecnologias (DGITS) no Ministério da Saúde, setor responsável pela Secretaria Executiva da CONITEC.

\section{Seleção dos critérios de filtragem e priorização}

Uma oficina de trabalho foi realizada em outubro de 2012 com o objetivo de discutir os critérios que serão utilizados nas etapas de filtragem e priorização de medicamentos novos e emergentes a serem prospectados pelo sistema de MHT.

A oficina de trabalho contou com a participação de 22 atores estratégicos da gestão de incorporação de tecnologias em saúde no SUS, representando diferentes órgãos do Ministério da Saúde, instituições acadêmicas, agências reguladoras, secretarias de estado de saúde e da CONITEC, principal cliente do sistema de MHT em construção.

Para o desenvolvimento das atividades, cinco grupos de trabalho foram formados, evitando-se a concentração de representantes de uma mesma instituição no mesmo grupo. Cada grupo pôde sugerir alterações na redação e descrição dos critérios de filtragem e priorização.

As atividades foram estruturadas em duas fases: seleção de critérios de filtragem e de priorização. Na seleção dos critérios de filtragem, foi solicitado que os atores participantes da oficina discutissem em grupo as definições dos quatro critérios propostos, avaliassem a condição final de filtragem e, se houvesse necessidade, sugerissem alguma mudança de inclusão e/ou exclusão de um novo critério.

A seleção dos critérios de priorização foi realizada em quatro etapas. Inicialmente, foi solicitado que cada participante selecionasse individualmente 10 critérios de maior importância. Após discussão das opiniões individuais no grupo, cada um dos grupos apresentou sua opinião. Finalmente, foram definidos como os critérios de consenso da oficina de trabalho os 10 critérios selecionados por pelo menos quatro dos cinco grupos de trabalho.

A última etapa foi a realização de uma nova reunião com um representante do DGITS, a fim de validar os critérios selecionados na oficina de trabalho que seriam utilizados para o sistema de MHT de medicamentos.
Análise das preferências dos atores

estratégicos na incorporação de

tecnologia em saúde

Para entender melhor o processo de seleção dos critérios e mapear as preferências dos atores, utilizou-se a análise de correspondência múltipla, que é uma das técnicas de análise multivariada utilizada para dados categóricos 8 .

A técnica propõe a redução do número de dimensões de uma matriz de dados facilitando a análise e a interpretação deles de forma gráfica, por meio do mapa de correspondência. Nesse mapa, são representados os atores que correspondem às linhas e os critérios que correspondem às colunas da matriz de dados, de modo a manter as relações existentes entre eles. Na análise do mapa, é possível verificar as associações existentes entre os indivíduos e os critérios com base na distância entre eles, portanto, quanto mais próximo, maior a probabilidade de associação. Além disso, é possível descrever as dimensões (eixos) do mapa em função das categorias dos critérios mais dispersos, que indicam um maior peso para aquela dimensão em questão ${ }^{8}$.

A seguir são descritos de maneira sucinta os passos da análise de correspondência múltipla ${ }^{8,9}$. A técnica é aplicada a uma matriz indicadora $\mathbf{Z}$, formada por códigos binários, em que nas linhas ( $i$ ) estão presentes os atores e nas colunas $(j)$ os critérios. Nessa matriz, cada critério é codificado com as categorias " 1 " se tiver sido selecionado pelo ator, ou “0” em caso contrário. A partir de Z, é realizada uma padronização por meio da equação 1 , formando a matriz de frequência relativa ou matriz de correspondência $\mathbf{F}_{\text {nxp }}$.

$f_{i j}=\frac{Z_{i j}}{T} \quad$ para $1 \leq i \leq n$ e $1 \leq j \leq p$

Em que:

$n=$ número de linhas (atores); $p=$ número de colunas (categorias dos critérios); $f_{i j}$ - corresponde à frequência relativa da resposta do ator $i$ a uma determinada categoria $j ; Z_{i j}$ - corresponde à resposta do ator $i$ a uma determinada categoria $j$ da matriz indicadora, podendo ter valores de 1 ou 0 ; $T$ - é o número total de elementos de $\mathbf{Z}$.

Em seguida, por meio da frequência relativa marginal de linhas $\left(\mathrm{r}_{\mathrm{i}}\right)$ e colunas $\left(\mathrm{c}_{\mathrm{j}}\right)$ é calculada a matriz de correspondência padronizada $\mathbf{G}_{\mathrm{nxp}}$, conforme a equação 2 .

$g_{\ddot{j}}=\frac{f_{j}}{\sqrt{r_{i}} \cdot \sqrt{c_{j}}}$ para $1 \leq i \leq n$ e $1 \leq j \leq p$

Em que: $g_{i j}$ - corresponde à frequência relativa padronizada da resposta do ator i a uma determinada categoria $\mathrm{j} ; f_{i j}$ - corresponde à frequência relativa da resposta do ator i a uma 
determinada categoria $\mathrm{j} ; r_{i}=\sum^{p} f_{j}-$ massa de linhas - é a soma das frequências da linha i da matriz de correspondência (frequência relativa marginal da linha i); $c_{j}=\sum_{i=1}^{n} f_{j}$ - massa de colunas - é a soma das frequências da coluna $\mathrm{j}$ da matriz de correspondência (frequência relativa marginal da coluna $\mathrm{j}$ ).

Em seguida, utiliza-se um algoritmo de decomposição por valores singulares ${ }^{8}$ para fatorar a matriz de correspondência padronizada $\mathbf{G}_{\mathrm{nxp}}$ em três diferentes matrizes. Duas matrizes de vetores singulares, sendo uma relativa aos atores $\left(\mathbf{U}_{\text {nxp }}\right)$ e outra relativa aos critérios $\left(\mathbf{V}_{\text {pxp }}\right)$. A terceira é uma matriz quadrada $\left(\mathbf{D}_{\mathrm{pxp}}\right)$, na qual a diagonal principal é composta por valores singulares associados a cada uma das dimensões. Essa matriz será utilizada para o cálculo da inércia que é a medida utilizada para indicar a variabilidade dos critérios no novo espaço dimensional (eixos) 8 .

$\mathrm{O}$ passo seguinte consiste em calcular as coordenadas-padrão de linha e coluna por meio da divisão das matrizes $\mathbf{U}_{\text {nxp }}$ e $\mathbf{V}_{\text {pxp }}$ pela raiz quadrada de suas massas de linha $\left(\mathrm{r}_{\mathrm{i}}\right)$ e coluna $\left(\mathrm{c}_{\mathrm{j}}\right)$, respectivamente. Como último passo, as coordenadas principais são calculadas pelo produto das coordenadas-padrão de linha e coluna, e seus respectivos valores singulares da matriz quadrada $\left(\mathbf{D}_{\text {pxp }}\right)$. As coordenadas principais contêm os valores das posições dos atores e das categorias dos critérios para cada uma das dimensões utilizadas para construir o mapa de correspondência. Desse modo, o Mapa de Correspondência é formado pela projeção das coordenadas principais de atores e/ou de categorias dos critérios nas duas ou três dimensões (eixos) de maior inércia (maior contribuição para a variabilidade dos dados) 8 .

A contribuição relativa para a inércia $\left(\mathrm{Ctr}_{\mathrm{jd}}\right)$ é uma medida da variância do eixo dado por uma determinada linha (ator) ou coluna (categoria do critério) do mapa, indicando a importância da linha ou coluna para certa dimensão. Essa contribuição 9 é calculada por meio da equação 3 . A contribuição relativa do critério é calculada como a soma das contribuições de suas categorias para a dimensão em análise 8,9.

$C t r_{j d}=\frac{c_{j} *\left(y_{j d}\right)^{2}}{\lambda_{d}}$

Em que: $c_{j}=$ frequência relativa marginal ou massa da categoria $j$; $y_{j d}=$ coordenada principal da categoria $j$ na dimensão $d ; \lambda_{\mathrm{d}}=$ inércia na dimensão $d$.

Foram considerados nesta análise os critérios de priorização inicialmente propostos na oficina de trabalho, exceto o critério "potencial impacto orçamentário no SUS" (O) por ter sido escolhido por todos os participantes da oficina de trabalho e assim não apresentar variabilidade entre eles. Os dois critérios "necessidade de capacitação da equipe" (CE) e "mudanças estruturais e/ou organizacionais no serviço" (ME) foram agregados ao critério "potencial impacto no custo para o serviço” (CS) após mudança durante a oficina. Foram utilizados na análise de correspondência múltipla os critérios finais com nova redação presentes na Tabela 1.

Todo o processamento foi realizado no programa estatístico R, versão 2.15.0, utilizando o pacote denominado correspondence analysis ca (The R Foundation for Statistical Computing, Viena, Áustria; http://www.r-project.org).

\section{Resultados}

Fase de preparação da oficina - identificação dos critérios

A busca pelos critérios resultou em um conjunto de 28 critérios utilizados em sistemas de MHT no mundo. Após a reunião com o representante da CONITEC, foi definida uma proposta de quatro critérios de filtragem e 19 critérios de priorização como base para ser apresentado e discutido nas atividades da oficina de trabalho.

Os critérios para filtragem apresentados na oficina foram: (1) horizonte de tempo entre a solicitação de pesquisa clínica e a finalização do processo de registro na ANVISA; (2) inovação ao atender as condições de tecnologia totalmente nova sem existir opção terapêutica, tecnologia com grande potencial de eficácia ou reduzidos efeitos adversos em relação às opções atuais e tecnologia com nova forma farmacêutica de apresentação; (3) relevância para as políticas de saúde prioritárias para o SUS; e (4) impacto epidemiológico da tecnologia. Na Tabela 1, são descritos os 19 critérios de priorização proposto na oficina de trabalho.

\section{Oficina de trabalho}

\section{- Critérios de filtragem}

As tecnologias filtradas deverão atender aos critérios de horizonte de tempo e inovação. Para o critério horizonte de tempo, as tecnologias deverão estar nas fases II ou III de pesquisa clínica, e, para a classe de medicamentos biológicos, tal prospecção deveria partir de uma fase anterior, a pré-clínica, segundo alguns atores. Para atender ao critério inovação, a tecnologia deverá ser classificada em uma das condições: totalmente nova sem opção terapêutica ou apresentar grande po- 
Critérios de priorização propostos e seleção final definida para o Monitoramento do Horizonte Tecnológico (MHT).

\begin{tabular}{|c|c|c|c|}
\hline Critérios propostos & $\begin{array}{l}\text { Seleção pelos } \\
\text { grupos }\end{array}$ & Critérios finais com nova redação & Sigla \\
\hline Tamanho do grupo elegível para utilizar a tecnologia & Todos & Relevância epidemiológica & REp \\
\hline $\begin{array}{l}\text { Relevância da tecnologia para as políticas de saúde prioritárias } \\
\text { para o SUS }\end{array}$ & Todos & Não houve mudança & Prio \\
\hline Impacto da tecnologia na mortalidade do grupo elegível & Todos & Não houve mudança & MT \\
\hline Existência de opção atual de tratamento para o grupo elegível & Todos & $\begin{array}{l}\text { Relevância da tecnologia no tratamento } \\
\text { atual do grupo elegível (inovação radical, } \\
\text { substitutiva ou complementar) }\end{array}$ & RTec \\
\hline Potencial impacto orçamentário no SUS & Todos & Não houve mudança & $\mathrm{O}$ \\
\hline Segurança & Todos & Não houve mudança & SG \\
\hline Potencial impacto no custo para o serviço (CS) & $A, B, E$ & & \\
\hline Necessidade de capacitação da equipe (CE) & $A, B, D, E$ & Potencial impacto no custo para o serviço & $\mathrm{CS}$ * \\
\hline Mudanças estruturais e/ou organizacionais no serviço (ME) & $A, B, D, E$ & & \\
\hline Aspectos legais, éticos e sociais & $B, C, D, E$ & Não houve mudança & LES \\
\hline Impacto da tecnologia na morbidade do grupo elegível & $B, E$ & - & $\mathrm{MB}$ \\
\hline Impacto da tecnologia na qualidade de vida no grupo elegível & $B, C, D$ & - & QV \\
\hline Impacto da tecnologia na sobrevida do paciente & $A, B, E$ & - & SB \\
\hline Impacto da tecnologia sobre o desempenho do serviço & B & - & DS \\
\hline Potencial para difusão inapropriada & C & - & $\mathrm{DI}$ \\
\hline Potencial impacto no custo para o paciente & B & - & $\mathrm{CP}$ \\
\hline Potencial impacto no custo para sociedade & $B, C$ & - & CSoc \\
\hline Mudanças na prática clínica & B & - & PC \\
\hline Robustez da evidência disponível & $A, D, E$ & - & $\mathrm{R}$ \\
\hline
\end{tabular}

* CS revisado: composto pelos três aspectos: custo para serviço, necessidade de capacitação e mudanças estruturais no serviço.

tencial de eficácia ou reduzidos efeitos adversos em relação às opções atuais.

\section{- Critérios de priorização}

Foram selecionados 10 dos 19 critérios propostos na oficina de trabalho. Na Tabela 1, são descritos os 19 critérios e os respectivos grupos que os escolheram com as sugestões de nova redação no nome do critério nos casos pertinentes. Foi sugerido que os critérios CE e ME deveriam ser agregados ao conceito do critério CS. Após discussão com o cliente, essa sugestão de mudança foi aceita e, desse modo, foram obtidos oito critérios finais para a etapa de priorização. São eles: "relevância epidemiológica" (REp), "relevância da tecnologia para as políticas de saúde prioritárias para o SUS" (Prio), "impacto da tecnologia na mortalidade do grupo elegível” (MT), "relevância da tecnologia no tratamento atual do grupo elegível” (inovação radical, substitutiva ou complementar) (RTec), critério O, "segurança" (SG), critério CS e "aspectos legais, éticos e sociais” (LES).
Mapeamento das preferências dos atores por meio de análise de correspondência múltipla

A Figura 1a ilustra o mapa de correspondência no qual são apresentadas as disposições dos critérios de priorização de acordo com as preferências dos indivíduos. Esse mapa é formado por um novo espaço dimensional composto pela primeira e a segunda dimensão ao invés das 16 dimensões, correspondentes aos 16 critérios analisados. Nessas duas novas dimensões, estão contidos 70,3\% da representatividade dos dados: a dimensão 1, eixo horizontal, representa $46,8 \%$ e a dimensão 2 , eixo vertical, representa $23,5 \%$. Os oito critérios de priorização finais selecionados na oficina de trabalho estão localizados na região à esquerda da dimensão 1 próximo à origem dos eixos.

Para melhorar a visualização do mapa, a região dos oito critérios selecionados da Figura la foi ampliada, de modo que as categorias " 1 " dos oito critérios finais selecionados pudessem ser mais bem visualizados (Figura 1b). 
1a) Visão geral

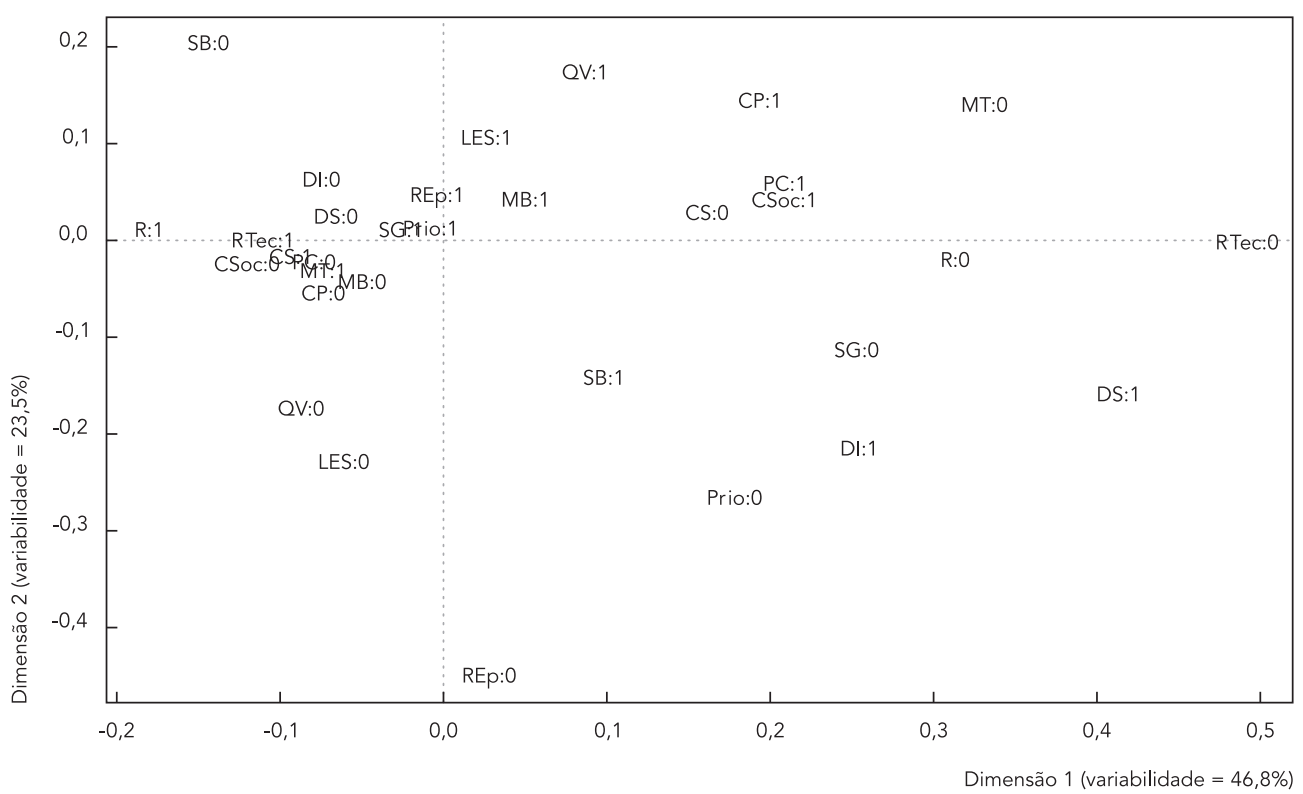

1b) Região ampliada da região à esquerda da dimensão 1 da Figura 1a

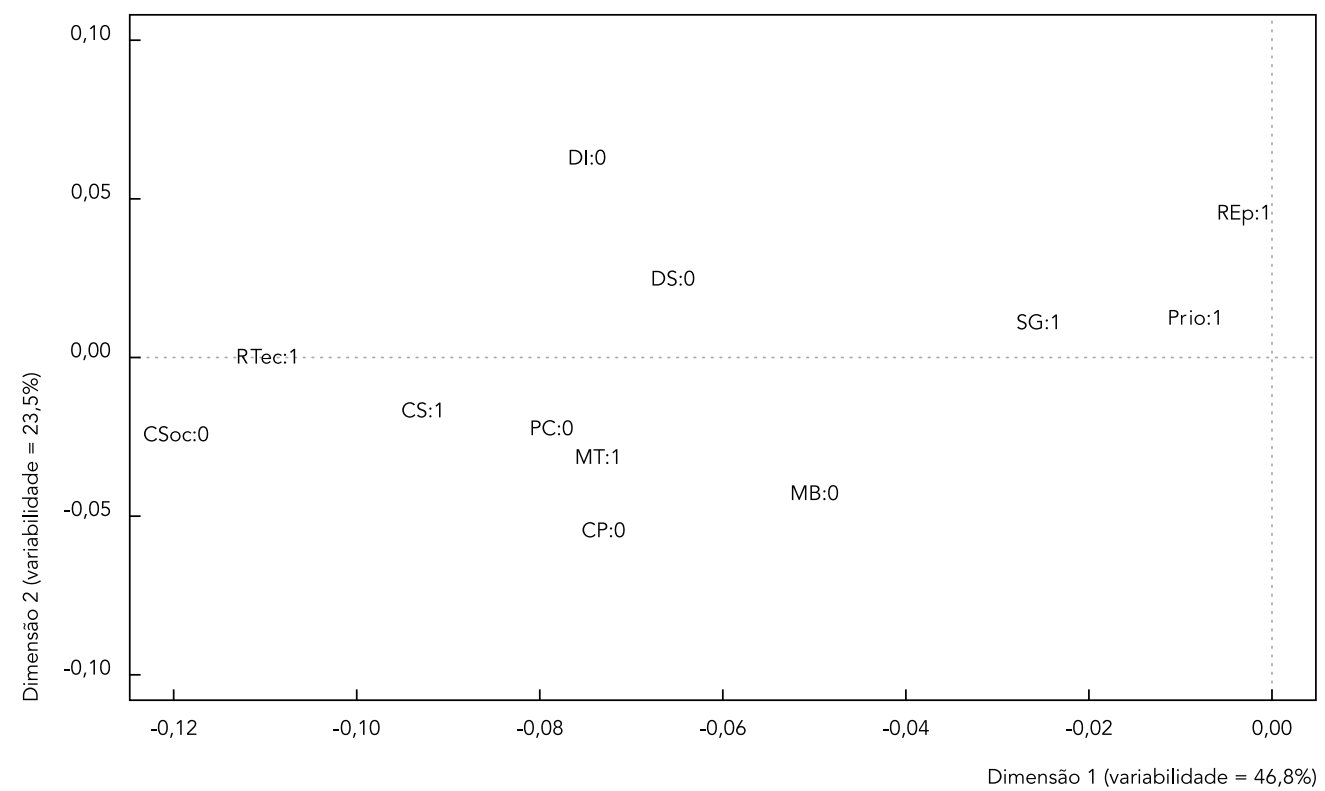

Critérios: REp: relevância epidemiológica; Prio: relevância da tecnologia para as políticas de saúde prioritárias para o SUS; MT: impacto da tecnologia na mortalidade do grupo elegível; RTec: relevância da tecnologia no tratamento atual do grupo elegível (inovação radical, substitutiva ou complementar); CS: potencial impacto no custo para o serviço; SG: Segurança; LES: aspectos legais, éticos e sociais; MB: impacto da tecnologia na morbidade do grupo elegível; QV: impacto da tecnologia na qualidade de vida no grupo elegível; SB: impacto da tecnologia na sobrevida do paciente; DS: impacto da tecnologia sobre o desempenho do serviço; DI: potencial para difusão inapropriada; CP: potencial impacto no custo para o paciente; CSoc: potencial impacto no custo para a sociedade; PC: mudanças na prática clínica; R: robustez da evidência disponível. 
Na dimensão 1 (Figura 1a), os critérios com maior dispersão, ou seja, com maior contribuição (inércia) para a formação da dimensão refletem questões relacionadas à evidência disponível e à relevância que a tecnologia possui. São eles: critério RTec, "robustez da evidência disponível” (R) e critério MT. À esquerda da dimensão 1 , esses critérios foram julgados como relevantes para a priorização (resposta 1 - selecionado). Em oposição, à direita da dimensão 1, foram julgados como irrelevantes para a priorização (resposta 0 - não selecionado).

Os valores de contribuição (inércia) de cada critério para a formação das dimensões 1 e 2, calculadas por meio da equação 3, estão apresentadas na Tabela 2. É possível observar que os critérios R (19,8\%), RTec (18,9\%) e MT (8,6\%) apresentam maiores contribuições para a dimensão 1, ratificando a importância deles na formação dessa dimensão.

Outro aspecto destacado na dimensão 1 é sobre os critérios relacionados a custo, em que se pode constatar que possuem disposição próxima um dos outros ("potencial impacto no custo pa- ra o paciente" - CP, "potencial impacto no custo para a sociedade” - CSoc e critério CS), e há uma divergência com relação a sua relevância para a priorização conforme o tipo de custo. À esquerda da dimensão 1, foi selecionado como relevante o "potencial impacto no custo para o serviço" (CS: 1). Em contrapartida, à direita, foi selecionado como relevante o "potencial impacto no custo para o paciente" (CP: 1) e "potencial impacto no custo para a sociedade" (CSoc: 1 ).

A análise da dimensão 2, vertical, sugere uma associação com critérios que representam impacto da tecnologia na vida do paciente, pois os critérios com maior dispersão nessa dimensão são: "impacto da tecnologia na sobrevida do paciente" (SB), "impacto da tecnologia na qualidade de vida no grupo elegível” (QV) e "aspectos legais, éticos e sociais” (LES). Os valores das contribuições desses critérios para a formação da dimensão 2 estão destacados na Tabela 2. Essa dimensão reflete um antagonismo desses critérios uma vez que na parte superior foi selecionado como relevante a QV e LES e em contrapartida, na parte inferior, foi selecionado o SB como relevante.

Tabela 2

Valores das contribuições dos critérios para a formação das dimensões 1 e 2 (inércia).

\begin{tabular}{|c|c|c|}
\hline \multirow[t]{2}{*}{ Critério } & \multicolumn{2}{|c|}{ Contribuição (inércia) para a formação da dimensão (\%) } \\
\hline & Dimensão 1 & Dimensão 2 \\
\hline REp & 0,0 & 14,3 \\
\hline Prio & 0,5 & 2,3 \\
\hline MT & 8,6 & 3,1 \\
\hline MB & 0,8 & 1,2 \\
\hline QV & 2,6 & 21,2 \\
\hline SB & 4,9 & 20,2 \\
\hline DS & 9,5 & 2,8 \\
\hline RTec & 18,9 & 0,0 \\
\hline $\mathrm{DI}$ & 6,7 & 9,5 \\
\hline $\mathrm{CP}$ & 5,0 & 5,5 \\
\hline CSoc & 8,8 & 0,7 \\
\hline $\mathrm{CS}$ & 5,2 & 0,3 \\
\hline PC & 5,8 & 0,9 \\
\hline $\mathrm{R}$ & 19,8 & 0,2 \\
\hline SG & 2,2 & 0,9 \\
\hline LES & 0,6 & 17,0 \\
\hline
\end{tabular}

CP: potencial impacto no custo para o paciente; CS: potencial impacto no custo para o serviço; DI: potencial para difusão inapropriada; CSoc: potencial impacto no custo para a sociedade; DS: impacto da tecnologia sobre o desempenho do serviço; LES: aspectos legais, éticos e sociais; MB: impacto da tecnologia na morbidade de grupo elegível; MT: impacto da tecnologia na mortalidade do grupo elegível; PC: mudança na prática clínica; Prio: relevância da tecnologia para as políticas de saúde prioritárias para o SUS; QV: impacto da tecnologia na qualidade de vida no grupo elegível; R: robustez da evidência disponível; REp: relevância epidemiológica; RTec: relevância da tecnologia no tratamento atual do grupo elegível (inovação radical, substitutiva ou complementar); SB: impacto da tecnologia na sobrevida do paciente; SG: segurança. 
A análise realizada acima sobre a dispersão das categorias dos critérios que se destacam nas duas dimensões pode ser utilizada para caracterizar as respectivas dimensões, entretanto é necessário observar que alguns critérios não puderam caracterizar as dimensões porque apresentam pouca variabilidade entre os indivíduos. Isso ocorreu para os critérios SG, REp, Prio e “impacto da tecnologia sobre o desempenho do serviço" (DS) que tiveram uma de suas categorias escolhidas por mais de $80 \%$ dos atores e estão localizadas proximamente à origem dos eixos. Assim, mesmo com as categorias complementares como SG:0, REp:0, Prio:0 e DS:1 apresentando maior dispersão sobre as dimensões 1 ou 2 (Figura 1a), os respectivos critérios não podem caracterizar essas dimensões.

O mapa de correspondência da Figura 2 apresenta a distribuição dos 22 atores participantes da oficina neste novo plano bidimensional, no qual se pode verificar que aproximadamente metade está agrupada próxima à região dos oito critérios finais de priorização, a esquerda da dimensão 1, e os outros estão distribuídos de modo aleatório nas direções opostas a essa região.
Uma análise da posição desses atores foi realizada considerando o grupo ao qual pertencia. Dessa maneira, constatou-se que os grupos A (participante 1 a 5) e D (participante 14 a 17) foram os grupos mais homogêneos, sendo que no grupo A um membro (participante 5) se distanciou dos outros. Os grupos A e D estão localizados na região em que se encontram os oito critérios finais de priorização selecionados. As características de ambos os grupos são similares com relação à dispersão sobre a dimensão 1 , com seus participantes localizados na região à esquerda da dimensão 1 caracterizada pela seleção dos critérios: RTec, R, MT e CS. Ao avaliar esses dois grupos pela perspectiva da dimensão 2, tal semelhança não existe, dado que os grupos se encontram em regiões opostas no mapa, de modo que o grupo A está localizado na região inferior representada pela seleção do critério SB. Em contrapartida, o grupo D está localizado na região superior à dimensão 2 , representada pela seleção dos critérios QV e LES.

Os demais grupos B (participante 6 a 9), C (participante 10 a 13) e E (participante 18 a 22) são mais heterogêneos entre si e se mostram

Figura 2

Mapa de correspondência dos atores participantes da seleção dos critérios de priorização para o Monitoramento do Horizonte Tecnológico (MHT).

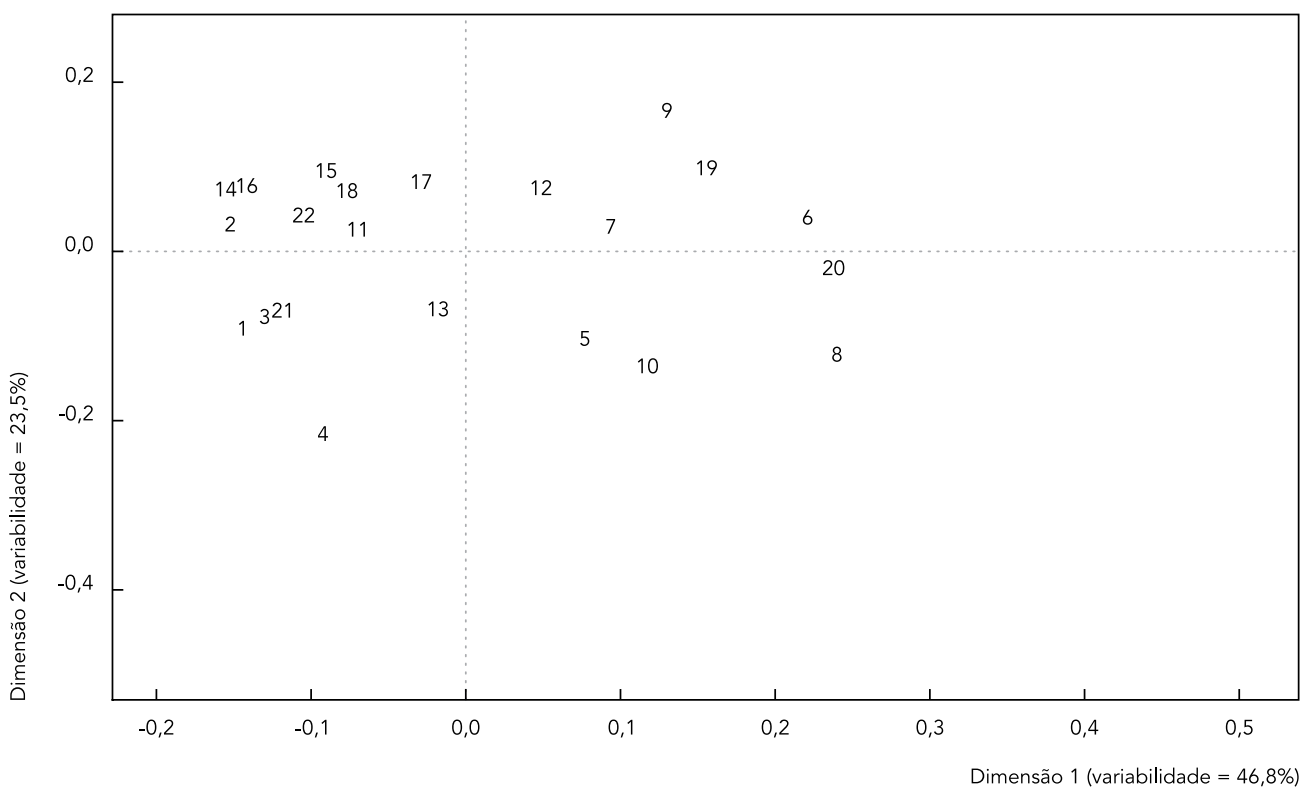

Grupo A: 1 a 5; Grupo B: 6 a 9; Grupo C: 10 a 13; Grupo D: 14 a 17; Grupo E: 18 a 22. 
dispersos com relação aos oito critérios finais de priorização. O grupo B apresenta maior espalhamento sobre a dimensão 2, exibindo opiniões internas contrárias para os critérios que caracterizam essa dimensão. Esse grupo se mostrou associado a critérios relacionados ao custo e aspectos da prática clínica como critério CSoc, critério $\mathrm{CP}$ e "mudança na prática clínica" (PC).

O grupo C, situado em torno da origem dos eixos, mostra uma contradição entre as opiniões dos atores sobre os critérios que caracterizam as dimensões 1 e 2, uma vez que estão situados em cada um dos quadrantes do mapa de correspondência. O grupo E apresenta maior espalhamento sobre a dimensão 1 , com seus participantes exibindo opiniões contrárias sobre os critérios que caracterizam essa dimensão. Alguns de seus participantes $(18,21$ e 22) possuem opiniões similares àquelas presentes nos grupos $\mathrm{A}$ e D (região à esquerda da dimensão 1) e outros (19 e 20) se assemelham às opiniões exibidas pelos participantes do grupo B (região à direita da dimensão 1).

\section{Discussão}

Diferentes autores 10,11,12 citam a importância da participação dos atores envolvidos na incorporação de tecnologias em saúde e de especialistas em ATS na elaboração de um sistema de MHT. Desse modo, a busca por critérios para as etapas de filtragem e priorização deveria não só envolver esses atores como também buscar entender o processo de estabelecimento dessas preferências, objetivando identificar eventuais conflitos de interesse, influência de especialistas ou formadores de opinião e formação profissional.

Nesse sentido, a metodologia proposta neste estudo possibilitou descrever o cenário de estabelecimento das preferências utilizando um mapa capaz de posicionar os atores de acordo com suas preferências pelos critérios e sua posição dentro do grupo, bem como a posição do grupo de acordo com os critérios.

Portanto, foi possível observar que os grupos A e D parecem ser mais homogêneos com relação às opiniões dos seus participantes e estão mais próximos ao conjunto dos oito critérios finais selecionados, sugerindo uma influência maior nessa escolha. Esses dois grupos possuem características semelhantes quanto à dimensão 1 (relacionada à evidência disponível e à relevância que a tecnologia possui), mas diferentes para a dimensão 2 (relacionada ao impacto que a tecnologia possa ter na vida do paciente), sendo o critério de sobrevida mais relevante para o grupo A e o de qualidade de vida mais relevante para o grupo D.
Por outro lado o grupo B e parte do grupo E consideraram mais relevantes os critérios relativos ao custo das tecnologias.

Foi ainda encontrada no grupo A a possível influência de um dos atores na opinião do grupo, em função da similaridade entre o perfil de preferência desse ator com o perfil do grupo. Essa influência parece ter ocorrido em função da experiência profissional desse ator. O mesmo não ocorreu para os demais grupos, que apresentaram maior heterogeneidade nas opiniões individuais para as duas dimensões. Dessa forma, não foi possível verificar a influência de outros fatores tais como a instituição ou conflito de interesse. Contudo, se atores externos ao SUS - tais como da indústria, de associação de pacientes dentre outros - estivessem envolvidos, os fatores acima poderiam ter sido observados. Isso mostra que a metodologia permite a participação de atores de diversos segmentos, o que torna o processo mais transparente, mas que, ao mesmo tempo, permite ao tomador de decisão entender essas influências.

Uma vez descrito o processo de definição das preferências quanto aos critérios, foi possível compará-los com os utilizados em sistemas de MHT já estabelecidos internacionalmente. Com relação aos critérios de filtragem (Inovação e Horizonte de Tempo), observou-se que eles também são utilizados por outros sistemas de MHT 10,13,14,15. Isso sugere que os atores têm preferência similar à observada em sistemas de MHT já estabelecidos em outros países. É importante ressaltar que os critérios de filtragem são determinantes para a seleção das tecnologias que serão monitoradas, pois para passar para a etapa de priorização, a tecnologia deve atender integralmente a ele, o que não ocorre com os critérios de priorização. Assim sendo, os critérios "ser uma inovação" e "estar em fase II ou III de pesquisa clínica” são indispensáveis para passar pela etapa de filtragem.

Diferente do critério de inovação que foi consenso entre os atores, o critério horizonte de tempo gerou discordância. Por exemplo, o grupo D, composto por atores com maior experiência em pesquisa clínica, destacou que incluir a fase II de pesquisa clínica no horizonte de tempo pode ser um problema, já que é grande o número de tecnologias em desenvolvimento na fase II que não atingem a fase III. Em uma consulta na base de dados Integrity (Thomson Reuters. https:// integrity.thomson-pharma.com/integrity/xmlx sl/, acessado em 17/Abr/2014) sobre as pesquisas clínicas em andamento, pode-se verificar que existe de fato maior número de tecnologias em desenvolvimento na fase II (44\%) do que na fase III (9\%) para o câncer de mama, por exemplo. 
Sobre esse aspecto, Nachtnebel et al. 10 relatam que o horizonte de tempo deve ser reduzido, pois se verificou que incluir a fase II o torna muito amplo. No processo de desenvolvimento do MHT para oncológicos da Áustria, esse critério foi redefinido passando a incluir somente a fase III de pesquisa clínica. Em contrapartida, Joppi et al. 13 relatam que na agência italiana, Italian Horizon Scanning Project (IHPS) estabelecida em 2006, além da fase III como horizonte de tempo é incluído a fase II para alguns medicamentos oncológicos. Essa abordagem foi utilizada por um período, sendo programada de acordo com os autores uma avaliação futura desse critério para adequar aos objetivos da agência.

No banco de dados sobre tecnologias emergentes, mantido pela EuroScan, existe uma predominância de tecnologias identificadas na fase III de pesquisa clínica, seguido pela fase em que a tecnologia já está estabelecida no mercado 16 . Isso pode sugerir que prospectar fases iniciais do desenvolvimento da tecnologia não é uma prática usual entre as agências de MHT uma vez que existe pouca evidência disponível nessas fases.

Desse modo, é possível identificar que existe uma divergência na literatura sobre qual horizonte de tempo utilizar, de acordo com a missão das agências e o objetivo do MHT. A mesma divergência foi encontrada no presente trabalho, o que pode ser um indicativo de que este critério venha a ser redefinido futuramente. Por hora, optou-se por mantê-lo como resultado da oficina.

No presente estudo, foram selecionados oito critérios de priorização dos quais cinco deles estão relacionados ao grupo elegível para utilizar a tecnologia (paciente). Paralelamente, esses oito critérios também são utilizados pelos membros da EuroScan, dos quais os mais usados são: REp, MT, CS e O. Por outro lado, os critérios menos utilizados são: LES, RTec e SG 14.

$\mathrm{Na}$ priorização específica de tecnologias novas na área oncológica, Nachtnebel et al. 10 descrevem que, em geral, cinco critérios são utilizados: número de pacientes elegíveis para a tecnologia sob consideração, intenção de uso da nova terapia (complementar ou substitutiva), estimado impacto dos benefícios clínicos e impacto estimado nos recursos financeiros. Desses cinco critérios, quatro deles são similares aos selecionados no presente trabalho, sugerindo que da mesma forma que na filtragem, os critérios de priorização selecionados pelos atores estão em consonância com os critérios utilizados pelos membros da EuroScan com experiência na prospecção de medicamentos oncológicos.

É possível observar que os membros da EuroScan utilizam critérios de priorização similares com pequenas diferenças, em razão, sobretudo, do papel da agência frente ao sistema de saúde. Além disso, segundo essas agências, alguns critérios, mesmo que importantes, podem ser inviáveis no processo de MHT em função da pouca informação disponível sobre a tecnologia 12. Logo, alguns dos critérios selecionados no presente estudo podem vir a ser alterados futuramente uma vez que, nessa fase do ciclo de vida da tecnologia, as informações são limitadas e geralmente associadas a incertezas.

Dos oito critérios de priorização selecionados, seis foram escolhidos por todos os grupos (conforme ilustrado na Tabela 1), indicando que ocorreu uma concordância entre os grupos sobre os critérios de maior relevância para a priorização. Por conseguinte, foi possível obter um conjunto de critérios com a aprovação de todos, mesmo sendo os atores membros de instituições com perfis de opinião diferentes.

No Brasil, assim como em outros países da América Latina, a ATS é utilizada para auxiliar as decisões de incorporação de tecnologias em saúde, entretanto a prospecção de novas tecnologias ainda está sendo desenvolvida, não existindo uma metodologia ou sistemática para antecipar as tecnologias que surgem no mercado ${ }^{17}$. Deste modo, os resultados desse estudo são relevantes para o processo de desenvolvimento de um sistema de MHT no país.

Dessa forma, espera-se que a abordagem metodológica de envolvimento dos atores estratégicos na seleção dos critérios, ajude a reduzir a possível resistência desses mesmos atores na implantação do sistema de MHT. A metodologia utilizada também permite que outros atores envolvidos ou não no atual processo de incorporação possam ser incluídos, tais como pesquisadores, pacientes, membros de conselhos de classe e indústria farmacêutica, de modo que se visualizem suas preferências e possíveis influências no processo de decisão.

O estudo demonstra a utilidade de se aplicar uma método de análise multivariada para visualizar o perfil de preferência dos atores estratégicos na incorporação de tecnologias em saúde e, desse modo, dar transparência ao processo de seleção dos critérios e apoiar o desenvolvimento de uma metodologia de prospecção de tecnologias relevantes ao SUS. 


\section{Colaboradores}

A. Nascimento contribuiu na pesquisa bibliográfica análise e interpretação de dados, redação e revisão do texto. A. T. Vidal e R. T. Almeida colaboraram na análise e interpretação de dados, redação e revisão do texto.

\section{Agradecimentos}

À Capes pelo financiamento com bolsa de doutorado, a Carta Acordo BR/LOA/1200120.001 - OPAS/OMSCOPPETEC e à Christina Carvalho Otto pelo apoio na oficina de trabalho.

\section{Referências}

1. Carlsson P, Jorgensen T. Scanning the horizon for emerging health technologies - conclusions from a European Workshop. Int J Technol Assess Health Care 1998; 14:695-704.

2. Douw K, Vondeling H. Selection of new health technologies for assessment aimed at informing decision making: a survey among horizon scanning systems. Int J Technol Assess Health Care 2006; 22:177-83.

3. Simpson S, Hiller J, Gutierrez-Ibarluzea I, Kearney B, Norderhaug I, Fay AF, et al. A toolkit for the identification and assessment of new and emerging health technologies. EuroScan Secretariat Department of Public Health, Epidemiology \& Biostatistics; 2009. http://euroscan.org.uk/mmlib/ includes/sendfile.php?id=24 (acessado em 01/ Out/2012).

4. EuroScan International Network. The international information network on new and emerging health technologies. http://euroscan.org.uk/tech nologies/member/all (acessado em 17/Set/2012).

5. Secretaria de Ciência, Tecnologia e Insumos Estratégicos, Ministério da Saúde. Monitoramento do horizonte tecnológico em saúde no âmbito da Rebrats: proposta preliminar; 2011. http://bvsms. saude.gov.br/bvs/publicacoes/monitoramen to_horizonte_tecnologico.pdf (acessado em 20/ Jan/2014)

6. Presidência da República. Decreto no 7646, de 21 de dezembro de 2001. Dispõe sobre a Comissão Nacional de Incorporação de Tecnologias no Sistema Único de Saúde e sobre o processo administrativo para incorporação, exclusão e alteração de tecnologias em saúde pelo Sistema Único de Saúde - SUS, e dá outras providências. http://www. planalto.gov.br/ccivil_03/_Ato2011-2014/2011/ Decreto/D7646.htm (acessado em 10/Jan/2014).

7. Packer C, Gutierrez-Ibarluzea I, Simpson S. The evolution of early awareness and alert methods and systems. Int J Technol Assess Health Care 2012; 28:199-200.
8. Greenacre M. Correspondence analysis in practice. 2nd Ed. Barcelona: Chapman and Hall/CRC; 2007.

9. Le Roux B, Rouanet H. Geometric data analysis from correspondence analysis to structured data analysis. Dordrecht: Kluwer Academic Publishers; 2004

10. Nachtnebel A, Geiger-Gritsch S, Hintringer K, Wild C. Scanning the horizon-development and implementation of an early awareness system for anticancer drugs in Austria. Health Policy 2012; 104:1-11.

11. Noorani HZ, Husereau DR, Boudreau R, Skidmore B. Priority setting for health technology assessments: a systematic review of current practical approaches. Int J Technol Assess Health Care 2007; 23:310-5.

12. Douw K, Vondeling H, Sørensen J, Jørgensen T, Sigmund $\mathrm{H}$. "The future should not take us by surprise": preparation of an early warning system in Denmark. Int J Technol Assess Health Care 2004; 20:342-50.

13. Joppi R, Demattè L, Menti AM, Pase D, Poggiani C, Mezzalira L. The Italian Horizon Scanning Project. Eur J Clin Pharmacol 2009; 65:775-81.

14. Gutierrez-Ibarluzea I, Simpson S, Benguria-Arrate G. Early awareness and alert systems: an overview of EuroScan methods. Int J Technol Assess Health Care 2012; 28:301-7.

15. Morrison A. Scanning the horizon in a decentralized healthcare system: the Canadian experience. Int J Technol Assess Health Care 2012; 28:327-32.

16. Ibargoyen-Roteta N, Gutierrez-Ibarluzea I, Benguria-Arrate G, Galnares-Cordero L, Asua J. Differences in the identification process for new and emerging health technologies: analysis of the EuroScan database. Int J Technol Assess Health Care 2009; 25:367-73.

17. Pichon-Riviere A, Elias FTS, Rivero, VG, Vaca CP. Early awareness and alert activities in Latin America: current situation in four countries. Int J Technol Assess Health Care 2013; 28:315-20. 


\section{Abstract}

Filtration and prioritization are two basics steps in horizon scanning systems. This article aimed to map stakeholders' preferences in the Brazilian Unified National Health System (SUS) regarding filtration and prioritization criteria. Two filtration criteria (time horizon and innovation) and eight prioritization criteria (relevance to epidemiology, health policies, and clinical practice; potential impact on SUS budget, healthcare providers' costs, and mortality; safety; and legal, ethical, and social aspects) were selected. Multiple correspondence analysis was used to map stakeholders preferences within and between groups. Two groups were more homogeneous and determinant for selection of prioritization criteria. Stakeholders' professional experience had more influence than institutional affiliations. The approach showed transparent criteria selection and analysis of stakeholders' individual preferences.

Biomedical Technology; Biomedical Technology Assessment; Health Planning

\section{Resumen}

Dentro de las etapas básicas de un sistema de evaluación precoz de tecnologías emergentes se encuentran las etapas de filtrado y priorización. Este trabajo tiene por objetivo mapear las preferencias de diferentes actores estratégicos del Sistema Único de Salud brasileno (SUS) para los criterios de filtrado y priorización. Se seleccionaron dos criterios de filtrado (horizonte de tiempo e innovación) y ocho criterios de priorización (relevancia: epidemiológica, en las políticas de salud y en la práctica clínica; impacto: en el presupuesto del SUS, en el costo para el servicio de salud y en la mortalidad; seguridad, aspectos legales, éticos y sociales). Se realizó un análisis de correspondencia múltiple para mapear las preferencias de los actores por grupo y entre grupos. Dos de los grupos fueron más homogéneos entre sí y determinantes para la selección de criterios de priorización. La influencia que tuvo la experiencia profesional fue más decisiva que la influencia institucional. Esta metodología permitió seleccionar criterios de forma transparente y analizar las preferencias individuales.

Tecnología Biomédica; Evaluación de la Tecnología Biomédica; Planificación en Salud
Recebido em 27/Nov/2014

Versão final reapresentada em 21/Mai/2015 Aprovado em 18/Fev/2016 